Gabriele Mondello

\title{
A remark on the homotopical dimension of some moduli spaces of stable Riemann surfaces
}

Received March 1, 2006 and in revised form October 21, 2006

\begin{abstract}
Using a result of Harer, we prove certain upper bounds for the homotopical/cohomological dimension of the moduli spaces of Riemann surfaces of compact type, of Riemann surfaces with rational tails and of Riemann surfaces with at most $k$ rational components. These bounds would follow from conjectures of Looijenga and Roth-Vakil.
\end{abstract}

\section{Introduction}

Tautological classes, Chow groups and rational cohomology of the moduli spaces of pointed Riemann surfaces have curious vanishing properties, which are not completely understood yet. It seems natural to look for a geometric explanation of these vanishings, namely these moduli spaces "should" be covered by a certain number of open affine sets, or at least "should" have an affine stratification with a certain number of strata. Notice that this number also bounds the maximal dimension of a complete subvariety contained in such a moduli space.

Historically, first Arbarello Arb74 realized that a stratification of the moduli space $\mathcal{M}_{g}$ of Riemann surfaces of genus $g$ could be useful to investigate the geometric properties of $\mathcal{M}_{g}$, starting from the locus of hyperelliptic curves and then climbing the $g-1$ layers of his stratification. Ten years later, Diaz [Dia84] used a variant of Arbarello's stratification to show that $\mathcal{M}_{g}$ cannot contain complete subvarieties of dimension $g-1$. Some years later, using similar stratifications, Looijenga [L0095] proved that the tautological classes in the Chow ring of $\mathcal{M}_{g}$ vanish in degree $\geq g-1$, which implies Diaz's result. He also showed that the tautological classes of the moduli space $\mathcal{M}_{g, n}^{\mathrm{rt}}$ of $n$-pointed stable Riemann surfaces of genus $g$ with rational tails vanish in degree $\geq g-1+n$. Then he formulated the following conjecture.

Conjecture 0.1 (Looijenga). Let $g, n \geq 0$ be such that $2 g-2+n>0$. The coarse space $M_{g, n}$ of $\mathcal{M}_{g, n}$ has a stratification

$$
M_{g, n}=\coprod_{i=1}^{g-\delta_{n, 0}} S_{i} \quad \text { with } \quad \bar{S}_{j}=\coprod_{i \leq j} S_{i} \quad \text { for all } j
$$

such that each locally closed stratum $S_{i}$ is affine.

G. Mondello: Department of Mathematics, Massachusetts Institute of Technology,

77 Massachusetts Avenue, Cambridge, MA 02139, USA; e-mail: gabriele@math.mit.edu 
The conjecture would be a consequence of the existence of a Zariski open cover of $\mathcal{M}_{g, n}$ made of $g-\delta_{n, 0}$ affines. On the other hand, one may notice that both Arbarello's and Diaz's stratifications of $\mathcal{M}_{g}$ have the right number of strata, while not even conjectural candidates have been proposed for the affines.

We stress that Conjecture 0.1 has much stronger consequences, as is clear from the following general statement.

Proposition 0.2. Let $M$ be a scheme of finite type of dimension $d$ over the field $\mathbb{K}$ and let $M=\coprod_{i=1}^{k} S_{i}$ be a stratification, with $\bar{S}_{j}=\coprod_{i \leq j} S_{i}$ for all $j$, such that each locally closed stratum $S_{i}$ is affine. Then $H_{\mathrm{ett}}^{r}(M ; \mathcal{F})=0$ for all $r>d+k-1$ and every constructible (or torsion, or l-adic) sheaf $\mathcal{F}$ on $M$. Moreover, if $M$ is reduced over $\mathbb{C}$, then the associated topological space $M^{\text {top }}$ is homotopy equivalent to a $C W$-complex of dimension at most $d+k-1$.

We refer to [RV04] for a proof of this proposition and for an extensive discussion on affine stratification and covering numbers and their applications to the moduli spaces of Riemann surfaces.

Looijenga's conjecture can be extended to other partial compactifications of the moduli space $\mathcal{M}_{g, n}$ of $n$-pointed Riemann surfaces of genus $g$. The most interesting cases are the moduli space $\mathcal{M}_{g, n}^{\mathrm{ct}}$ of stable Riemann surfaces of compact type (i.e. whose dual graph is a tree), the moduli space $\mathcal{M}_{g, n}^{\mathrm{rt}}$ of stable Riemann surfaces with rational tails, the moduli space $\mathcal{M}_{g, n}^{\mathrm{irr}}$ of irreducible stable Riemann surfaces and the moduli space $\mathcal{M}_{g, n}^{\leq k}$ of stable Riemann surfaces with at most $k$ rational components.

The interest for the last moduli space $\mathcal{M}_{g}^{\leq k}$, is related to a vanishing result due to Ionel [Ion02] in cohomology and to Graber and Vakil [GV05] in the Chow ring: it says that the tautological classes of $\mathcal{M}_{g} \leq k$ vanish in degree $\geq g+k$, which implies Looijenga's vanishing and Getzler's conjecture [Get98] (which says that $\psi_{i_{1}} \cdots \psi_{i_{g}}=0$ on $\mathcal{M}_{g, n}$ ). As a consequence of these investigations, Roth and Vakil [RV04] wondered about the meaning of this new stratification of $\overline{\mathcal{M}}_{g, n}$ by number of rational components and they raised the following question.

Conjecture 0.3 (Roth-Vakil). Let $g, n>0$ be such that $2 g-2+n>0$. The coarse space of $\mathcal{M}_{g, n}^{\leq k}$ (resp. $\mathcal{M}_{g, n}^{\mathrm{rt}}, \mathcal{M}_{g, n}^{\mathrm{ct}}$ ) has an affine stratification made of $g+k$ (resp. $g+n-1,2 g-2+n)$ strata.

The purpose of the present paper is to analyze the homotopy type of these moduli spaces of Riemann surfaces, proving as a corollary that their rational cohomological dimension is what we would expect if Conjecture 0.3 were true, according to Proposition 0.2 . Hence, in a certain sense, our results support Conjecture 0.3

Our computations heavily rely on the case of smooth Riemann surfaces, which was investigated by Harer [Har86] in the following way.

The moduli space $\mathcal{M}_{g, n}$ is the quotient of the Teichmüller space $\mathcal{T}_{g, n}$ by the action of the mapping class group $\Gamma_{g, n}$. As $\mathcal{T}_{g, n}$ is homeomorphic to a Euclidean space and $\Gamma_{g, n}$ acts with finite stabilizers, the orbifold-theoretic quotient $\mathcal{M}_{g, n}$ represents $B \Gamma_{g, n}$. Hence, the cohomology groups of $\mathcal{M}_{g, n}$ are those of $\Gamma_{g, n}$ essentially by definition; so, instead 
of dealing with the orbifold $\mathcal{M}_{g, n}$, Harer could work $\Gamma_{g, n}$-equivariantly on $\mathcal{T}_{g, n}$. In particular, he found a $\Gamma_{g, n}$-equivariant retraction of $\mathcal{T}_{g, n}$ onto a subcomplex of dimension $4 g-4+n$ when $g, n>0$ or $n-3$ if $g=0$, thus bounding the homotopical dimension of every unramified cover of $\mathcal{M}_{g, n}$ which is a manifold, and so the virtual cohomological dimension of $\Gamma_{g, n}$. Actually, he also proved that $\Gamma_{g, n}$ is a virtual duality group and so that the bound is sharp.

However, dealing with partial compactifications of $\mathcal{M}_{g, n}$ which are not $K(G, 1)$ 's, we cannot directly reduce to equivariantly work on some partial compactification of the Teichmüller space but we would need to put our hands on the orbifolds themselves. In fact, it turns out that some objects we are interested in are simply connected, as shown in Appendix Appendix A Moreover, we would need a virtual (that is, orbifold) analogue of homotopical and cohomological dimension of an orbispace.

Instead of treating these technicalities in full detail, we prefer to show how the main result works for the moduli spaces of Riemann surfaces with level structures $\mathcal{M}_{g, n}^{\lambda}$ whose Deligne-Mumford compactification $\overline{\mathcal{M}}_{g, n}^{\lambda}$ is a smooth variety, sketching the needed modifications to make the argument work for moduli spaces of Riemann surfaces which are orbifolds.

We incidentally remark that the cohomology with rational coefficients of any orbifold coincides with that of its coarse space and that the virtual homotopical dimension of an orbifold actually bounds the homotopical dimension of its coarse space, so that one can also read our result just at the coarse level, with little loss of information.

Our main result (Theorem 2.6 concerns any partial compactification of the moduli space of Riemann surfaces obtained by adding some strata of its Deligne-Mumford compactification. For the partial compactifications mentioned above, it reduces to the following.

Corollary 0.4. Let $g \geq 0$ and $2 g-2+n>0$. The moduli spaces of Riemann surfaces which are irreducible, of compact type, with rational tails or with at most $k$ rational components have the following upper bounds on their virtual homotopical/cohomological dimension.

\begin{tabular}{ccc}
\hline $\begin{array}{c}\text { Moduli space of stable } \\
\text { Riemann surfaces }\end{array}$ & $\begin{array}{c}\text { Virtual homotopical } \\
\text { dimension for } n>0\end{array}$ & $\begin{array}{c}\text { Virtual cohomological } \\
\text { dimension for } n=0\end{array}$ \\
\hline $\mathcal{M}_{g, n}^{\mathrm{irr}}$ & $4 g-3+n$ & $4 g-3$ \\
$\mathcal{M}_{g, n}^{\mathrm{ct}}$ & $5 g-6+2 n$ & $5 g-6$ \\
$\mathcal{M}_{g, n}^{\mathrm{rt}}$ with $g>0$ & $4 g-5+2 n$ & $4 g-5$ \\
$\mathcal{M}_{g, n}^{\leq k}$ & $4 g-4+n+k$ & $4 g-4+k$
\end{tabular}

The idea is the following: given a smooth complex variety $X$, a divisor $D \subset X$ with normal crossings induces a locally closed stratification by smooth subvarieties. One can reconstruct the homotopy type of $X$ from the homotopy type of the strata and of natural torus bundles over the strata (see Proposition 1.1p. Thus, if one knows the homotopical (resp. cohomological) dimension of each stratum, then one can produce an upper bound for the homotopical (resp. cohomological) dimension of $X$ (see Corollary 1.3. 
Deligne-Mumford's compactification $\overline{\mathcal{M}}_{g, n}$ has a natural divisor with normal crossings (in the orbifold sense) $\partial \mathcal{M}_{g, n}$, that is, the locus of singular surfaces. Each stratum $\mathcal{M}_{\sigma}$ of the induced stratification parametrizes stable Riemann surfaces of topological type $\sigma$.

The beauty of Deligne-Mumford's compactification is that all open strata $\mathcal{M}_{\sigma}$ are isomorphic to products of smaller moduli spaces of smooth Riemann surfaces, possibly up to the action of a finite group of symmetries. Hence, Harer's result allows us to conclude.

We would like to stress that the particular role played by rational components, which was noticed by Graber and Vakil, is already present in Harer's result. In fact, for $n>0$, the virtual homotopical dimension of $\mathcal{M}_{g, n}$ is $4 g-4+n$ if $g>0$ but $n-3$ (and not $n-4$ but one more!) if $g=0$. This shift appears to be responsible, at least at a topological level, for the homotopical dimension to grow according to the number of rational components.

\section{Smooth varieties and divisors with normal crossings}

Let $X$ be a smooth complex variety of dimension $N$ and $D$ a divisor with simple ${ }^{1}$ normal crossings, that is, $D=\bigcup_{i \in I} \bar{D}_{i}$ such that $\bar{D}_{i}$ is a smooth divisor of $X$ and any two components $\bar{D}_{i}$ and $\bar{D}_{j}$ intersect transversely. For every $J \subset I$, set $\bar{D}_{J}:=\bigcap_{j \in J} \bar{D}_{j}$ and identify $X$ with $\bar{D}_{\emptyset}$.

We will denote by $\widehat{D}_{J}$ the manifold with corners obtained by performing a real oriented blow-up $b_{J}: \widehat{D}_{J} \rightarrow \bar{D}_{J}$ along to $\bigcup_{i \in I \backslash J} \bar{D}_{J \cup\{i\}}$. We will also write $\widehat{X}$ for $\widehat{D}_{\emptyset}$. Notice that the natural inclusion $D_{J}:=\bar{D}_{J} \backslash \bigcup_{J \subsetneq L} \bar{D}_{L} \hookrightarrow \widehat{D}_{J}$ is a homotopy equivalence.

For every chain $J_{0} \subsetneq J_{1} \subsetneq \cdots \subsetneq J_{k} \subseteq I$, define $\widehat{D}_{J_{0}, \ldots, J_{k}}:=\widehat{D}_{J_{0}} \times \bar{D}_{J_{0}} \bar{D}_{J_{k}}$ and $D_{J_{0}, \ldots, J_{k}}:=\widehat{D}_{J_{0}} \times \bar{D}_{J_{0}} D_{J_{k}}$. The inclusion $D_{J_{0}, \ldots, J_{k}} \hookrightarrow \widehat{D}_{J_{0}, \ldots, J_{k}}$ is a homotopy equivalence and $\widehat{D}_{J_{0}, \ldots, J_{k}} \rightarrow \widehat{D}_{J_{k}}$ can be identified to the $\left(S^{1}\right)^{d}$-bundle

$$
\prod_{i \in J_{k} \backslash J_{0}} b_{J_{k}}^{*}\left(\left.S N\left(\bar{D}_{i} / X\right)\right|_{\bar{D}_{J_{k}}}\right)
$$

where $S N\left(\bar{D}_{i} / X\right)$ is the $S^{1}$-bundle associated to the normal bundle of $\bar{D}_{i}$ and $d=$ $\left|J_{k} \backslash J_{0}\right|$.

Now, define the simplicial topological space (without degeneracies) $X_{\bullet}$ as $X_{k}:=$ $\bigsqcup_{J_{0} \subsetneq \ldots \subsetneq J_{k}} \widehat{D}_{J_{0}, \ldots, J_{k}}$, with the following face maps. For $l=1, \ldots, k-1$, the face map $f_{l}: \widehat{D}_{J_{0}, \ldots, J_{k}} \rightarrow \widehat{D}_{J_{0}, \ldots, \widehat{J}_{l}, \ldots, J_{k}}$ is the identity. The face map $f_{k}: \widehat{D}_{J_{0}, \ldots, J_{k}} \rightarrow \widehat{D}_{J_{0}, \ldots, J_{k-1}}$ is induced by the inclusion $\bar{D}_{J_{k}} \hookrightarrow \bar{D}_{J_{k-1}}$, whereas $f_{0}: \widehat{D}_{J_{0}, \ldots, J_{k}} \rightarrow \widehat{D}_{J_{1}, \ldots, J_{k}}$ is induced by the projection

$$
\left.\left.\prod_{i \in J_{k} \backslash J_{0}} S N\left(\bar{D}_{i} / X\right)\right|_{\bar{D}_{J_{k}}} \rightarrow \prod_{i \in J_{k} \backslash J_{1}} S N\left(\bar{D}_{i} / X\right)\right|_{\bar{D}_{J_{k}}} .
$$

\footnotetext{
1 The simplicity of $D$ is not necessary but the notation becomes lighter in this case.
} 
Proposition 1.1. There is a natural homotopy equivalence between $X$ and the topological realization $\left|X_{\bullet}\right| \simeq \underset{\longleftarrow}{\text { hocolim }} X_{\bullet}$.

Let us recall that the topological realization $\left|X_{\bullet}\right|$ of a simplicial topological space $X_{\bullet}$ is obtained from $\bigsqcup_{i>0} X_{i} \times \Delta_{i}$ by identifying $\left(x_{i}, t_{0}, \ldots, t_{k-1}, 0, t_{k+1}, \ldots, t_{i}\right) \in X_{i} \times \Delta_{i}$ with $\left(f_{k}\left(x_{i}\right), t_{0}, \ldots, t_{k-1}, t_{k+1}, \ldots, t_{i}\right) \in X_{i-1} \times \Delta_{i-1}$ for every $i \geq 1$ and $0 \leq k \leq i$.

Proof. Let $\mathcal{I}_{d} \subset \mathcal{P}(I)$ be the set of subsets $J \subset I$ of cardinality $N-d$ such that $\bar{D}_{J}$ is nonempty and let $\mathcal{I}=\bigcup_{d} \mathcal{I}_{d}$.

Set $X(-1):=X$ and proceed by induction on $d$.

Let $0 \leq d \leq N$ and suppose we have already defined $X(r)$ and $V_{J}$ with $J \in \mathcal{I}_{r}$ for all $0 \leq r<d$. For every $J \in \mathcal{I}_{d}$ choose a tubular neighborhood $V_{J}$ of $\bar{D}_{J} \cap X(d-1)$ inside $X(d-1)$ in such a way that the closures of all $V_{J}$ 's inside $X$ are disjoint. Let $X(d):=X(d-1) \backslash \bigcup_{J \in \mathcal{I}_{d}} V_{J}$.

Define the open cover $\mathcal{U}=\left\{U_{J} \mid J \in \mathcal{I}\right\}$ of $X$, with $U_{J}$ consisting of a small fattening of $\bar{V}_{J}$ inside $X$, in such a way that:

- every $U_{J}$ retracts by deformation onto $V_{J}$,

- $U_{J} \cap U_{K}$ is nonempty if and only if $J \subseteq K$ or $K \subseteq J$,

- for every chain $J_{0} \subsetneq J_{1} \subsetneq \cdots \subsetneq J_{k}$ the natural map $U_{J_{0}} \cap \cdots \cap U_{J_{k}} \stackrel{\simeq}{\rightarrow} D_{J_{0}, \ldots, J_{k}}$ is a homotopy equivalence.

As the realization of a simplicial topological space represents the homotopy colimit of the associated system, the morphism $U_{\bullet} \rightarrow X_{\bullet}$ defined above induces a homotopy equivalence $\left|U_{\bullet}\right| \stackrel{\simeq}{\longrightarrow}\left|X_{\bullet}\right|$.

By the Čech-Segal construction [Seg68], every partition of unity on $X$ subordinated to the open cover $\mathcal{U}$ gives a homotopy equivalence $X \rightarrow\left|U_{\bullet}\right|$.

Remark 1.2. If $D$ still has normal crossings but some components of $D$ self-intersect, then the result above holds with minor (but obvious, and mostly notational) modifications.

The homotopical dimension $\operatorname{htdim}(X)$ of a topological space $X$ is the smallest dimension of a CW-complex that is homotopically equivalent to $X$. The cohomological dimension $\operatorname{chldim}(X)$ of $X$ is the smallest $k \in \mathbb{N}$ such that $H^{n}(X ; \mathbb{L})=0$ for every $n>k$ and every local system $\mathbb{L}$ over $X$. Clearly, $\operatorname{htdim}(X) \geq \operatorname{chldim}(X)$.

Corollary 1.3. Let $X$ be a complex manifold and $D$ a divisor with normal crossings.

(a) The homotopical dimension $\operatorname{htdim}(X)$ is bounded above by the maximum of $\operatorname{htdim}\left(D_{J}\right)+2|J|$ for $J \subseteq I$.

(b) The cohomological dimension chldim $(X)$ is bounded above the maximum of $\operatorname{chldim}\left(D_{J}\right)+2|J|$ for $J \subseteq I$.

Proof. As $\left|X_{\bullet}\right|$ is a quotient of $\bigsqcup_{k} \Delta_{k} \times X_{k}$, the space $X$ is homotopy equivalent to a CWcomplex made of cells of dimension at most $\max \left\{k+\operatorname{htdim}\left(X_{k}\right) \mid X_{k} \neq \emptyset\right\}$. Moreover, 
$\operatorname{htdim}\left(X_{k}\right) \leq \operatorname{htdim}\left(D_{J_{0}, \ldots, J_{k}}\right) \leq\left|J_{k}\right|-\left|J_{0}\right|+\operatorname{htdim}\left(D_{J_{k}}\right)$ and the maximum of $k+\left|J_{k}\right|-$ $\left|J_{0}\right|+\operatorname{htdim}\left(D_{J_{k}}\right)$ is attained when $\left|J_{k}\right|=k$ and $\left|J_{0}\right|=0$.

For the cohomological dimension, consider the open cover $\mathcal{U}$ as in the proof of Proposition 1.1 (with minor but obvious modifications if some components of $D$ self-intersect). Given a local system $\mathbb{L}$ on $X$, we can build a Leray spectral sequence associated to the open cover $\mathcal{U}$ of $X$ with $E_{1}$-term

$$
E_{1}^{p, q}=\bigoplus_{J_{0} \subsetneq \cdots \subsetneq J_{p} \in \mathcal{I}} H^{q}\left(U_{J_{0}} \cap \cdots \cap U_{J_{p}} ; \mathbb{L}\right)
$$

converging to $H^{p+q}(X ; \mathbb{L})$, where every component of $d_{1}$ is induced by the natural inclusion. The same computation as above gives the desired upper bound.

Remark 1.4. An orbifold $X$ has virtual homotopical dimension $\leq d$ if $X$ is homotopy equivalent to an orbisimplicial complex (that is, a complex of groups) of dimension $d$. Moreover, $X$ has virtual cohomological dimension $\leq d$ if $H^{k}(X ; \mathbb{L})=0$ for all $k>d$ and all local systems $\mathbb{L}$ on $X$. Statements analogous to Proposition 1.1 and Corollary 1.3 hold in the orbifold category.

\section{Moduli spaces of Riemann surfaces}

Let $g$ and $n$ be nonnegative integers such that $2 g-2+n>0$. Consider the moduli space $\mathcal{M}_{g, n}$ of Riemann surfaces $\Sigma$ of genus $g$ together with $n$ distinct points $\left\{p_{1}, \ldots, p_{n}\right\}$ $\hookrightarrow \Sigma$.

Let $\overline{\mathcal{M}}_{g, n}$ be the Deligne-Mumford compactification of $\mathcal{M}_{g, n}$, obtained by adding Riemann surfaces $\Sigma$ with nodes such that the marked points sit in the smooth locus of $\Sigma$ and every rational component of $\Sigma$ contains at least three special points, that is, points which are either marked or nodes.

Both moduli space $\mathcal{M}_{g, n}$ and $\overline{\mathcal{M}}_{g, n}$ are orbifolds and $\overline{\mathcal{M}}_{g, n}$ is compact. The boundary $\partial \mathcal{M}_{g, n}:=\overline{\mathcal{M}}_{g, n} \backslash \mathcal{M}_{g, n}$ is a normal crossing divisor in the orbifold sense.

Definition 2.1. Let $\mathcal{B}_{g, n}$ be the set of all homeomorphism classes of stable n-pointed Riemann surfaces of (arithmetic) genus $g$. For every $\sigma \in \mathcal{B}_{g, n}$ the stratum $\mathcal{M}_{\sigma}$ of $\overline{\mathcal{M}}_{g, n}$ is the locally closed suborbifold of Riemann surfaces that belong to $\sigma$.

A nonempty open set of strata of $\overline{\mathcal{M}}_{g, n}$ is a nonempty subset $\mathcal{S} \subseteq \mathcal{B}_{g, n}$ that satisfies the following condition: if $\sigma \in \mathcal{S}$ is obtained from $\sigma^{\prime} \in \mathcal{B}_{g, n}$ by pinching some simple closed curves of $\sigma^{\prime}$ to nodes, then $\sigma^{\prime} \in \mathcal{S}$.

A nonempty open set of strata $\mathcal{S} \subseteq \mathcal{B}_{g, n}$ determines a partial compactification $\overline{\mathcal{M}}_{\mathcal{S}}:=$ $\bigcup_{\sigma \in \mathcal{S}} \mathcal{M}_{\sigma}$ of $\mathcal{M}_{g, n}$ made of strata of $\overline{\mathcal{M}}_{g, n}$ and this correspondence is bijective. Clearly, $\mathcal{B}_{g, n}$ corresponds to $\overline{\mathcal{M}}_{g, n}$ and the trivial system consisting of the smooth surfaces only corresponds to $\mathcal{M}_{g, n}$. 
Example 2.2. The moduli spaces $\mathcal{M}_{g, n}^{\mathrm{irr}}$ of irreducible Riemann surfaces, $\mathcal{M}_{g, n}^{\mathrm{rt}}$ of Riemann surfaces with rational tails (that is, with an irreducible component of geometric genus $g$ ), $\mathcal{M}_{g, n}^{\leq k}$ of stable Riemann surfaces with at most $k$ rational components, and $\mathcal{M}_{g, n}^{\text {ct }}$ of Riemann surfaces of compact type (that is, whose dual graph is a tree) are partial compactifications of $\mathcal{M}_{g, n}$ of the type described above.

We would like to apply Corollary 1.3 to $\overline{\mathcal{M}}_{\mathcal{S}}$ with $\mathcal{S}$ a nonempty open set of strata, but $\overline{\mathcal{M}}_{\mathcal{S}}$ is usually an orbifold and not a manifold. To get around this problem we could extend the treatment of Section 1 to orbifolds (see Remark 1.4), which is very natural but requires some technicalities.

Instead, we will treat the case of moduli spaces of Riemann surfaces with finite level structures $\mathcal{M}_{g, n}^{\lambda}$ which are smooth manifolds and admit a smooth compactification $\overline{\mathcal{M}}_{g, n}^{\lambda}$, and we will sketch how to deduce the result for $\overline{\mathcal{M}}_{\mathcal{S}}$.

We denote by $\mathcal{M}_{g, n}^{\lambda} \rightarrow \mathcal{M}_{g, n}$ the covering space associated to a finite index subgroup $\Gamma^{\lambda} \subset \Gamma_{g, n}=\pi_{0} \operatorname{Diff}_{+}\left(\Sigma,\left\{p_{1}, \ldots, p_{n}\right\}\right)$. It is known by the work of Serre that $\mathcal{M}_{g, n}^{\lambda}$ is a manifold if $\Gamma^{\lambda}$ acts trivially on $H^{1}(\Sigma ; \mathbb{Z} / \ell \mathbb{Z})$ for some $\ell \geq 3$. It follows from Looijenga [Loo94], Pikaart-de Jong [PdJ95] and Boggi-Pikaart [BP00] that there exist finite index subgroups $\Gamma^{\lambda} \subset \Gamma_{g, n}$ such that the normalization $\overline{\mathcal{M}}_{g, n}^{\lambda}$ of $\overline{\mathcal{M}}_{g, n}$ in the field of functions of $\mathcal{M}_{g, n}^{\lambda}$ is a smooth manifold. We refer to these papers and to [DM69] for a detailed treatment of level structures, and to [ACV03] for a modular interpretation of the compactified spaces.

Fix a subgroup $\Gamma^{\lambda} \subset \Gamma_{g, n}$ of finite index such that $\overline{\mathcal{M}}_{g, n}^{\lambda}$ is a smooth manifold. The boundary $\partial \mathcal{M}_{g, n}^{\lambda}:=\overline{\mathcal{M}}_{g, n}^{\lambda} \backslash \mathcal{M}_{g, n}^{\lambda}$ is a divisor with normal crossings and the map $f^{\lambda}: \overline{\mathcal{M}}_{g, n}^{\lambda} \rightarrow \overline{\mathcal{M}}_{g, n}$ is a finite ramified covering.

A stratum of $\overline{\mathcal{M}}_{g, n}^{\lambda}$ is an irreducible component of $\left(f^{\lambda}\right)^{-1}\left(\mathcal{M}_{\sigma}\right)$, where $\sigma \in \mathcal{B}_{g, n}$. This defines a natural map $\mathcal{B}_{g, n}^{\lambda} \ni \tilde{\sigma} \mapsto[\tilde{\sigma}] \in \mathcal{B}_{g, n}$, where $\mathcal{B}_{g, n}^{\lambda}=\left\{\tilde{\sigma} \mid \mathcal{M}_{\tilde{\sigma}}\right.$ is a stratum of $\overline{\mathcal{M}}_{g, n}^{\lambda}$. An open set of strata $\mathcal{S}^{\lambda} \subseteq \mathcal{B}_{g, n}^{\lambda}$ is a nonempty subset such that $\overline{\mathcal{M}}_{\mathcal{S}^{\lambda}}:=$ $\bigcup_{\tilde{\sigma} \in \mathcal{S}^{\lambda}} \mathcal{M}_{\tilde{\sigma}}$ is open inside $\overline{\mathcal{M}}_{g, n}^{\lambda}$.

The first important fact is that the restriction $\mathcal{M}_{\tilde{\sigma}} \rightarrow \mathcal{M}_{[\tilde{\sigma}]}$ is a finite unramified cover. The second (much more) important fact is Harer's fundamental result.

Theorem 2.3 ([|[Har86] $)$. Let $h, m \geq 0$ be such that $2 h-2+m>0$. There exists $a \Gamma_{h, m^{-}}$ equivariant deformation retraction of the Teichmüller space $\mathcal{T}_{h, m}$ onto a $C W$-complex of dimension $4 h-4+m$ if $h, m>0$ and of dimension $m-3$ if $h=0$ and $m \geq 3$. If $m=0$ and $h \geq 2$, then $\Gamma_{h}$ has virtual cohomological dimension $4 h-5$.

We are now ready to prove the following.

Theorem 2.4. Let $g, n \geq 0$ be such that $2 g-2+n>0$ and let $\Gamma^{\lambda} \subset \Gamma_{g, n}$ be a finite index subgroup such that $\overline{\mathcal{M}}_{g, n}^{\lambda}$ is a smooth manifold. For every nonempty open set of strata $\mathcal{S}^{\lambda} \subseteq \mathcal{B}_{g, n}^{\lambda}$ : 
- if $n>0$, then the moduli space $\overline{\mathcal{M}}_{\mathcal{S}^{\lambda}}$ has homotopical dimension at most $4 g-4+n+$ $\operatorname{rc}\left(\mathcal{S}^{\lambda}\right)$,

- if $n=0$ and $\overline{\mathcal{M}}_{\mathcal{S}^{\lambda}} \neq \mathcal{M}_{g}^{\lambda}$, then the moduli space $\overline{\mathcal{M}}_{\mathcal{S}^{\lambda}}$ has cohomological dimension at most $4 g-4+n+\operatorname{rc}\left(\mathcal{S}^{\lambda}\right)$,

where $\operatorname{rc}\left(\mathcal{S}^{\lambda}\right)$ is the maximum number of rational components occurring in $[\tilde{\sigma}]$ for all $\tilde{\sigma} \in \mathcal{S}^{\lambda}$.

Proof. The homotopical dimension of $\mathcal{M}_{\tilde{\sigma}}$ can be easily computed using Harer's result, because $\mathcal{M}_{\tilde{\sigma}}$ is a finite unramified cover of $\mathcal{M}_{[\tilde{\sigma}]}$, and $\mathcal{M}_{[\tilde{\sigma}]}$ looks like $\prod_{i} \mathcal{M}_{h_{i}, m_{i}}$ modulo a finite group of symmetries: for instance, one can proceed inductively on the codimension of the stratum. The upshot is that, if $\tilde{\sigma}$ has at least a node or a puncture, then $\operatorname{htdim}\left(\mathcal{M}_{\tilde{\sigma}}\right)=4 g-4+n-2 v(\tilde{\sigma})+\operatorname{rc}(\tilde{\sigma})$, where $v(\tilde{\sigma})$ is the number of nodes of $[\tilde{\sigma}]$ and $\operatorname{rc}(\tilde{\sigma})$ is the number of rational components of $[\tilde{\sigma}]$. The result follows from Corollary 1.3

Remark 2.5. Notice that $\widehat{\mathcal{M}_{g, n}^{\lambda}}$ is the quotient $\widehat{T}_{g, n} / \Gamma^{\lambda}$ of Harvey's bordification $\widehat{T}_{g, n}$ of the Teichmüller space (see [Har81]).

To deduce a similar result for $\overline{\mathcal{M}}_{\mathcal{S}}$ (or for any orbifold $\overline{\mathcal{M}}_{\mathcal{S}^{\mu}}$, corresponding to a finite level structure $\mu$ ) we could invoke Remark 1.4

At the very end, the whole thing boils down to the following. Let $\Gamma^{\mu} \subseteq \Gamma_{g, n}$ be a finite index subgroup and let $\mathcal{S}^{\mu} \subseteq \mathcal{B}_{g, n}^{\mu}$ be a nonempty open set of strata. Pick a finite index subgroup $\Gamma^{\lambda} \subset \Gamma^{\mu}$ such that $\overline{\mathcal{M}}_{g, n}^{\lambda}$ is a smooth manifold and let $\mathcal{S}^{\lambda}$ be the set of strata of $\overline{\mathcal{M}}_{g, n}^{\lambda}$ that map to $\overline{\mathcal{M}}_{\mathcal{S}^{\mu}}$ through the natural map $\overline{\mathcal{M}}_{g, n}^{\lambda} \rightarrow \overline{\mathcal{M}}_{g, n}^{\mu}$.

Proposition 1.1 applied to $X=\overline{\mathcal{M}}_{\mathcal{S}^{\lambda}}$ and $D=\overline{\mathcal{M}}_{\mathcal{S}^{\lambda}} \cap \partial \mathcal{M}_{g, n}^{\lambda}$ tells us that $\overline{\mathcal{M}}_{\mathcal{S}^{\lambda}}$ is homotopy equivalent to $\left|\left(\overline{\mathcal{M}}_{\mathcal{S}^{\lambda}}\right)_{\bullet}\right|$. The result for $\overline{\mathcal{M}}_{\mathcal{S}^{\mu}}$ holds (on replacing "dimensions" by "virtual dimensions") if we can arrange this homotopy equivalence to be $\Gamma^{\mu}$-equivariant.

This can be done by just pulling back the open cover $\mathcal{U}$ of $\overline{\mathcal{M}}_{\mathcal{S}^{\lambda}}$ and the partition of unity subordinated to $\mathcal{U}$ in the proof of Proposition 1.1 from their analogues on $\overline{\mathcal{M}}_{\mathcal{S}^{\mu}}$.

Furthermore, one can observe that the singularities of any finite $\overline{\mathcal{M}}_{g, n}^{\lambda}$ are well-behaved with respect to the stratification induced by $\partial \mathcal{M}_{g, n}^{\lambda}$, so that we can even drop the requirement of $\overline{\mathcal{M}}_{g, n}^{\lambda}$ being an orbifold.

Hence, we can refine the statement of Theorem 2.4 .

Theorem 2.6. Let $g, n \geq 0$ be such that $2 g-2+n>0$. Let $\Gamma^{\mu} \subset \Gamma_{g, n}$ be a finite index subgroup and $\mathcal{S}^{\mu} \subseteq \mathcal{B}_{g, n}^{\mu}$ a nonempty set of strata such that $\overline{\mathcal{M}}_{\mathcal{S}^{\mu}}$ is open in $\overline{\mathcal{M}}_{g, n}^{\mu}$. Then:

- if $n>0$, then the moduli space $\overline{\mathcal{M}}_{\mathcal{S}^{\mu}}$ has virtual homotopical dimension at most $4 g-4+n+\operatorname{rc}\left(\mathcal{S}^{\mu}\right)$

- if $n=0$ and $\overline{\mathcal{M}}_{\mathcal{S}^{\mu}} \neq \mathcal{M}_{g}^{\mu}$, then the moduli space $\overline{\mathcal{M}}_{\mathcal{S}^{\mu}}$ has virtual cohomological dimension at most $4 g-4+n+\mathrm{rc}\left(\mathcal{S}^{\mu}\right)$.

Corollary 2.7. Let $g \geq 0$ and $2 g-2+n>0$. The moduli spaces of Riemann surfaces which are irreducible, of compact type, with rational tails or with at most $k$ rational components have the following upper bounds on their virtual homotopical/cohomological dimension. 


\begin{tabular}{ccc}
\hline $\begin{array}{c}\text { Moduli space of stable } \\
\text { Riemann surfaces }\end{array}$ & $\begin{array}{c}\text { Virtual homotopical } \\
\text { dimension for } n>0\end{array}$ & $\begin{array}{c}\text { Virtual cohomological } \\
\text { dimension for } n=0\end{array}$ \\
\hline $\mathcal{M}_{g, n}^{\mathrm{irr}}$ & $4 g-3+n$ & $4 g-3$ \\
$\mathcal{M}_{g, n}^{\mathrm{ct}}$ & $5 g-6+2 n$ & $5 g-6$ \\
$\mathcal{M}_{g, n}^{\mathrm{rt}}$ with $g>0$ & $4 g-5+2 n$ & $4 g-5$ \\
$\mathcal{M}_{g, n}^{\leq k}$ & $4 g-4+n+k$ & $4 g-4+k$ \\
\hline
\end{tabular}

The same result for Riemann surfaces with rational tails could be obtained in a simpler way. In fact, it is trivial for $g=0$ and it reduces to Harer's result for $n=0,1$. For $g>0$ and $n>1$, one can notice that the forgetful map $\mathcal{M}_{g, n}^{\mathrm{rt}} \rightarrow \mathcal{M}_{g, 1}^{\mathrm{rt}}$ is a fiber bundle, whose fiber is a smooth manifold of (real) dimension $2(n-1)$.

Finally, one can also check that for $\overline{\mathcal{M}}_{g, n}$ one gets the correct bound.

\section{Appendix A. The fundamental group of some moduli spaces}

In his fundamental paper [Har86] on the virtual cohomological dimension of the mapping class group, Harer was interested in moduli spaces which are $K(G, 1)$ 's. In that case, our definition of virtual homotopical (resp. cohomological) dimension reduces to the homotopical (resp. cohomological) dimension of the classifying space of a torsionfree subgroup of $G$ of finite index. However, if our moduli spaces are not $K(G, 1)$ 's, then this clearer and simpler definition is no longer available. In this short appendix we check that most partial compactifications of $\mathcal{M}_{g, n}$ we are interested in are not $K(G, 1)$ 's.

Proposition A.1. The moduli space $\mathcal{M}_{g, n}^{\mathrm{rt}}$ of Riemann surfaces with rational tails is a $K(G, 1)$ only for $(g, n)$ equal to $(0,3)$ or $(g, 0),(g, 1),(g, 2)$ and $g>0$.

Proof. In genus 0 , we have $\mathcal{M}_{0, n}^{\mathrm{rt}}=\overline{\mathcal{M}}_{0, n}$ so that it is always simply connected and never a $K(G, 1)$ (except in the trivial case $n=3$ ). For $g>0$, we know that $\mathcal{M}_{g, n}^{\text {rt }}=\mathcal{M}_{g, n}$ for $n=0,1$ and also that $\mathcal{M}_{g, 2}^{\mathrm{rt}}$ is the universal curve over $\mathcal{M}_{g, 1}$. For $n>2$ there is a topological fibration $F \rightarrow \mathcal{M}_{g, n}^{\mathrm{rt}} \rightarrow \mathcal{M}_{g, 1}$. Consider $[S, p] \in \mathcal{M}_{g, 1}$ and let $F_{[S, p]}$ be the fiber over $[S, p]$. Then $F_{[S, p]}$ is a projective variety of complex dimension $n-1$. If $n>2$, then $F_{[S, p]}$ contains a rational curve, so that $\pi_{2}(F) \neq 0$ and $\pi_{2}\left(\mathcal{M}_{g, n}^{\mathrm{rt}}\right) \neq 0$.

Proposition A.2. The moduli space $\mathcal{M}_{g, n}^{\mathrm{irr}}$ of irreducible stable Riemann surfaces is a $K(G, 1)$ only if $g=0$ (when it coincides with $\left.\mathcal{M}_{0, n}\right)$ and it is simply connected otherwise.

Proof. Trivially $\mathcal{M}_{0, n}^{\mathrm{irr}}=\mathcal{M}_{0, n}$. Instead, for $g \geq 1$ the mapping class group $\Gamma_{g, n}$ is generated by Dehn twists around nondisconnecting simple closed curves, so that $\mathcal{M}_{g, n}^{\text {irr }}$ is simply connected. However, $H^{2}\left(\mathcal{M}_{g, n}^{\mathrm{irr}} ; \mathbb{Q}\right)$ is not zero for $g>0$ (see [AC98]).

Proposition A.3. The moduli space $\mathcal{M}_{g}^{\leq k}$, of stable Riemann surfaces with at most $k$ rational components is a $K(G, 1)$ only for $(g, n, k)$ equal to $(0, n, 1)$ (when it coincides with $\left.\mathcal{M}_{0, n}\right)$ and $(1, n, 0)$ (when it coincides with $\left.\mathcal{M}_{1, n}\right)$ and it is simply connected otherwise. 
Proof. The genus 0 case is trivial because $\mathcal{M}_{0, n}^{\leq 0}$ is empty, $\mathcal{M}_{0, n}^{\leq 1}$ is equal to $\mathcal{M}_{0, n}$ and $\mathcal{M}_{0, n}^{\leq k}$ is simply connected for $k \geq 2$, but $H^{2}\left(\mathcal{M}_{0, n}^{\leq k} ; \mathbb{Q}\right) \neq 0$ for $k \geq 2$. For $g=1$ and $k=0$ we have $\mathcal{M}_{1, n}^{\leq 0}=\mathcal{M}_{1, n}$. Instead, for $g=1$ and $k \geq 1$ or for $g>1$ and $k \geq 0$ the moduli space $\mathcal{M}_{g, n}^{\leq k}$ is simply connected because $\Gamma_{g, n}$ is generated by Dehn twists around nondisconnecting curves. One can check that in all these cases $H^{2}\left(\mathcal{M}_{g, n}^{\leq k} ; \mathbb{Q}\right)$ does not vanish.

Proposition A.4. The moduli space $\mathcal{M}_{g, n}^{\mathrm{ct}}$ of stable Riemann surfaces of compact type is a $K(G, 1)$ only for $(g, n)$ equal to $(0,3),(1,1),(1,2),(2,0)$.

Proof. $\mathcal{M}_{0, n}^{\mathrm{ct}}$ is $\overline{\mathcal{M}}_{0, n}$ and $\mathcal{M}_{1, n}^{\mathrm{ct}}$ is exactly $\mathcal{M}_{1, n}^{\mathrm{rt}}$. An exceptional case is $\mathcal{M}_{2,0}^{\mathrm{ct}}$ which coincides with $\mathcal{A}_{2}$, the moduli space of principally polarized Abelian varieties of dimension 2. For $(g, n)$ greater than or equal to $(2,2),(3,1)$ or $(4,0)$ the moduli spaces $\mathcal{M}_{g, n}^{\text {ct }}$ contain a complete rational curve, so that $\pi_{2}\left(\mathcal{M}_{g, n}^{\text {ct }}\right) \neq 0$. For $(g, n)=(2,1),(3,0)$, one can notice that the maps into Deligne's torsors (see [HL97]) $\mathcal{M}_{2,1}^{\text {ct }} \rightarrow \mathcal{D}_{2}$ and $\mathcal{M}_{3,0}^{\text {ct }} \rightarrow \mathcal{D}_{3}^{p r}$ induce isomorphisms on fundamental groups, but not on rational cohomology. Moreover $\mathcal{D}_{g}$ and $\mathcal{D}_{g}^{\mathrm{pr}}$ are $K(G, 1)$ 's. So $\mathcal{M}_{g, n}^{\mathrm{ct}}$ is a $K(G, 1)$ only for $(g, n)$ equal to $(0,3),(1,1),(1,2)$ and $(2,0)$.

Acknowledgments. I would like to thank Enrico Arbarello, John Harer, Jason Starr and Ravi Vakil for valuable discussions on moduli spaces of Riemann surfaces, and Mark Behrens and André Henriques for useful conversations on the Čech-Segal construction and orbispaces. I also thank the referee for suggesting the use of level structures and Harvey's bordification to simplify the argument and for useful comments.

\section{References}

[AC98] Arbarello, E., Cornalba, M.: Calculating cohomology groups of moduli spaces of curves via algebraic geometry. Inst. Hautes Études Sci. Publ. Math. 88, 97-127 (1999) Zbl 0991.14012 MR 1733327

[ACV03] Abramovich, D., Corti, A., Vistoli, A.: Twisted bundles and admissible covers. Comm. Algebra 31, 3547-3618 (2003) Zbl 1077.14034 MR 2007376

[Arb74] Arbarello, E.: Weierstrass points and moduli of curves. Compos. Math. 29, 325-342 (1974) Zbl 0355.14013 MR 0360601

[BP00] Boggi, M., Pikaart, M.: Galois covers of moduli of curves. Compos. Math. 120, 171-191 (2000) Zbl 0959.14010 MR 1739177

[DM69] Deligne, P., Mumford, D.: The irreducibility of the space of curves of given genus. Inst. Hautes Études Sci. Publ. Math. 36, 75-109 (1969) Zbl 0181.48803 MR 0262240

[Dia84] Diaz, S.: A bound on the dimensions of complete subvarieties of $\mathcal{M}_{g}$. Duke Math. J. 51, 405-408 (1984) Zbl 0581.14017 MR 0747872

[Get98] Getzler, E.: Topological recursion relations in genus 2. In: Integrable Systems and Algebraic Geometry (Kobe/Kyoto, 1997), World Sci., River Edge, NJ, 73-106 (1998) Zbl 1021.81056 MR 1672112 
[GV05] Graber, T., Vakil, R.: Relative virtual localization and vanishing of tautological classes on moduli spaces of curves. Duke Math. J. 130, 1-37 (2005) Zbl pre05004321 MR 2176546

[HL97] Hain, R., Looijenga, E.: Mapping class groups and moduli spaces of curves. In: Algebraic Geometry (Santa Cruz 1995), Proc. Sympos. Pure Math. 62, Amer. Math. Soc., Providence, RI, 97-142 (1997) Zbl 0914.14013 MR 1492535

[Har86] Harer, J. L.: The virtual cohomological dimension of the mapping class group of an orientable surface. Invent. Math. 84, 157-176 (1986) Zbl 0592.57009 MR 0830043

[Har81] Harvey, W. J.: Boundary structure of the modular group. In: Riemann Surfaces and Related Topics (Stony Brook, NY, 1978) (Princeton, N.J.), Ann. of Math. Stud. 97, Princeton Univ. Press, 245-251 (1981) Zbl 0461.30036 MR 0624817

[Ion02] Ionel, E.-N.: Topological recursive relations in $H^{2 g}\left(\mathcal{M}_{g, n}\right)$. Invent. Math. 148, 627-658 (2002) Zbl 1056.14076 MR 1908062

[Loo94] Looijenga, E.: Smooth Deligne-Mumford compactifications by means of Prym level structures. J. Algebraic Geom. 3, 283-293 (1994) Zbl 0814.14030 MR 1257324

[Loo95] Looijenga, E.: On the tautological ring of $\mathcal{M}_{g}$. Invent. Math. 121, 411-419 (1995) Zbl 0851.14017 MR 1346214

[PdJ95] Pikaart, M., de Jong, A. J.: Moduli of curves with non-abelian level structure. In: The Moduli Space of Curves (Texel Island, 1994), Progr. Math. 129, Birkhäuser Boston, Boston, MA, 1995, 483-509 (1995) Zbl 0860.14024 MR 1363068

[RV04] Roth, M., Vakil, R.: The affine stratification number and the moduli space of curves. In: Algebraic Structures and Moduli Spaces, CRM Proc. Lecture Notes 38, Amer. Math. Soc., Providence, RI, 213-227 (2004) Zbl 1070.14021 MR 2096147

[Seg68] Segal, G.: Classifying spaces and spectral sequences. Inst. Hautes Études Sci. Publ. Math. 34, 105-112 (1968) Zbl 0199.26404 MR 0232393 